\title{
Heme oxygenase-1 retards hepatocellular carcinoma progression through the microRNA pathway
}

\author{
CHAOXIA ZOU ${ }^{1 *}$, CHENDAN ZOU $^{1 *}$, WANPENG CHENG ${ }^{1}$, ${\text { QIANG } \text { LI }^{3}, \text { ZHONGJING HAN }^{5}, \text { XIAONA WANG }}^{1}$, \\ JIANFENG JIN $^{1}$, JIAQI ZOU ${ }^{2}$, ZHIYAN LIU ${ }^{2}$, ZHONGQIU ZHOU ${ }^{2}$, WEIMING ZHAO ${ }^{1}$ and ZHIMIN DU ${ }^{2,4}$ \\ ${ }^{1}$ Department of Biochemistry and Molecular Biology, Harbin Medical University, Harbin, Heilongjiang; \\ ${ }^{2}$ Institute of Clinical Pharmacy and ${ }^{3}$ Department of General Surgery, The Second Affiliated Hospital of \\ Harbin Medical University, Harbin, Heilongjiang; ${ }^{4}$ The University Key Laboratory of Drug Research of \\ Heilongjiang Province, Harbin, Heilongjiang; ${ }^{5}$ Department of Hemopathology, \\ General Hospital of Daqing Oil Field, Daqing, Heilongjiang, P.R. China
}

Received April 5, 2016; Accepted July 25, 2016

DOI: $10.3892 /$ or.2016.5056

\begin{abstract}
Heme metabolism system is involved in microRNA (miRNA) biogenesis. The complicated interplay between heme oxygenase-1 (HO-1) and miRNA has been observed in various tissues and diseases, including human malignancy. In the present study, our data showed that stable HO-1 overexpression in hepatocellular carcinoma (HCC) cells downregulated several oncomiRs. The most stably downregulated are miR-30d and miR-107. Iron, one of HO-1 catalytic products, was an important mediator in this regulation. Cell function analysis demonstrated that HO-1 inhibited the proliferation and metastasis of HepG 2 cells, whereas miR-30d/miR-107 improved the proliferative and migratory ability of HepG 2 cells. The beneficial effect of HO-1 in HCC inhibition could be reversed by upregulating miR-30d and miR-107. Akt and ERK pathways may be involved in the regulation of HO-1/miR-30d/miR-107 in HCC. These data indicate that HO-1 significantly suppresses HCC progression by regulating the miR-30d/miR-107 level, suggesting $\mathrm{miR}-30 \mathrm{~d} / \mathrm{miR}-107$ regulation as a new molecular mechanism of HO-1 anticancer effect.
\end{abstract}

Correspondence to: Professor Zhimin Du, Institute of Clinical Pharmacy, The Second Affiliated Hospital of Harbin Medical University, 246 Baojian Road, Nangang, Harbin, Heilongjiang, P.R. China

E-mail: duzhimin2016@tom.com

Professor Weimin Zhao, Department of Biochemistry and Molecular Biology, Harbin Medical University, 157 Baojian Road, Nangang, Harbin, Heilongjiang, P.R. China

E-mail: zhaowm1969@126.com

*Contributed equally

Key words: heme oxygenase-1, miRNAs, hepatocellular carcinoma, proliferation, metastasis

\section{Introduction}

Heme oxygenase-1 (HO-1) is an inducible enzyme that degrades heme to carbon monoxide, ferrous ions and biliverdin (1). Accumulating evidence supports that HO-1 could modulate the tumor growth and metastasis through its regulation of apoptosis, angiogenesis and inflammatory $(2,3)$. Moreover, compared with the surrounding healthy tissues, HO-1 expression is increased in various tumors, such as glioblastoma, melanoma (4) and hepatocellular carcinoma (5), and decreased in non-small cell lung carcinoma (6). Previous study also confirmed that HO-1 inhibited the migratory ability of hepatocellular carcinoma (HCC) cells $(3,7)$. However, recent studies demonstrated that HO-1 plays a contradictory role in several types of malignancies including breast, lung and prostate cancer (8-10). The molecular mechanism of HO-1 in HCC still require elucidating.

MicroRNAs (miRNAs), a class of small non-coding RNAs, is one of the most abundant classes of gene regulatory molecules in eukaryotic cells (11). miRNAs participate in various biological processes of tumor progression including proliferation, migration and angiogenesis by causing translational repression or degradation of the mRNA. It is not surprising that HMOXI expression can be regulated by miRNAs. It has been reported that HO-1 protein level could be downregulated by miR-122 or miR-217/377 (12-14). Furthermore, Kozakowska et al (15) revealed that both specific miRNA expressions and global miRNA biogenesis could be also regulated by HO-1. These studies implied that HO-1 plays a complicated role by modulating miRNA. Moreover, the regulatory role of $\mathrm{HO}-1$ on miRNA is tissue-specific. The relation between HO-1 and miRNAs remains unknown in HCC.

In the present study, our results showed that overexpressing HO-1 could reduce the expression of both miR-30d and miR-107. Furthermore, HO-1 could repress the proliferation and migration of $\mathrm{HCC}$ both in vivo and in vivo which depends on reducing the expression of miR-30d and miR-107. Iron, one of HO-1 enzymatic products, plays an important role in suppressing the expression of miR-30d and miR-107. During the regulation of proliferation and migration, 
Akt and ERK pathways may be involved in the function of HO-1/miR-30d/miR-107 in HCC.

\section{Materials and methods}

Cell culture, treatment and drug preparation. The human hepatocellular cells HepG2 were maintained in Dulbecco's modified Eagle's medium that was supplemented with $10 \%$ fetal bovine serum (FBS), $100 \mathrm{U} / \mathrm{ml}$ penicillin $\mathrm{G}$ and $100 \mu \mathrm{g} / \mathrm{ml}$ streptomycin at $37^{\circ} \mathrm{C}$ in a humidified incubator containing $5 \% \mathrm{CO}_{2}$. Cobalt protoporphyrin IX (CoPPIX), tin protoporphyrin IX (SnPPIX), bilirubin and tricarbonyldichlororuth enium(II) dimer (CORM-2) (Sigma, Shanghai, China) stock solutions were prepared by dissolving in dimethyl sulfoxide at a concentration of $20 \mu \mathrm{g} / \mathrm{l}$ of stock solution. Ferricitrate and deferoxamine were respectively dissolved in deionized water at concentration of $20 \mathrm{mM}$. iCORM is an inactive form of CORM-2.

Cell transfection. HepG2 cells were seeded at a density of $2 \times 10^{5}$ cells in a 6-well plate and grown to $60-70 \%$ confluency in growth media. Cells were transfected with pcDNA3.1 (+) containing human wild-type HO-1 (HepG2/HO-1) and empty vector (HepG2/Mock) using Lipofectamine 2000 transfection reagent (Invitrogen, Carlsbad, CA, USA). The stable cell lines were selected with $500 \mu \mathrm{g} / \mathrm{ml} \mathrm{G} 418$ (Sigma) and screened for HO-1 protein expression. For gene silencing, the pLL3.7, pLL3.7-HO-1shRNA ( $4 \mu \mathrm{g})$, that were kindly provided by Professor Hong Zhou (Academy of Military Medical Sciences, Beijing, China), were used. miR-30d and miR-107 mimics (and their Nc mimics) (100 pmol) (GenePharma, Shanghai, China) were transiently transfected into HepG2/HO-1 or HepG2/Mock cells using Lipofectamine 2000 transfection reagent. After $24 \mathrm{~h}$, the transfected cells were used for further experiments. H1B and Mock were previously described (3).

Cell viability assay. Cell viability was determined by 3-(4,5-dimethylthiazol-2-yl)-2.5-diphenyl-tetrazolium bromide (MTT) assay as previously described (3).

Cell migration assay. To detect the ability of cells to migrate in vivo, we used the Transwell chamber assay. Briefly, HepG2 cells $\left(5 \times 10^{4}\right)$ were placed in the upper compartment of a 24 -well Transwell unit with $8 \mu \mathrm{m}$ polycarbonate nucleopore filters (Corning Costar, Cambridge, MA, USA). Medium containing $10 \%$ FBS was added to the lower compartment. The cells were then incubated for $24 \mathrm{~h}$ in a humidified atmosphere of $5 \% \mathrm{CO}_{2}$ at $37^{\circ} \mathrm{C}$. The cells were then fixed and counted as previously described (3).

Colony formation assay. HepG2/HO-1 and HepG2/Mock cells, and HepG2 cells that were transfected with miR-30d, miR-107 and NC mimics were seeded in $3.5-\mathrm{cm}$ dishes ( 1,000 cells/dish) and cultured for 2 weeks to allow for colony formation. The colonies were fixed in methanol, stained with $0.1 \%$ crystal violet and counted.

Real-time quantitative polymerase chain reaction (qRT-PCR). Total RNA was isolated using TRIzol reagent (Invitrogen) according to the manufacturer's protocol, and the concentration of total RNA was measured with a NanoDrop 2000c. RNA $(1 \mu \mathrm{g})$ was converted to cDNA using miR-30d- and miR-107-specific stem-loop primer, and the cDNA and qRT-PCR with miR-30d- and miR-107-specific primers was performed using a 7500 Real-Time PCR system (Applied Biosystems, Mannheim, Germany). For relative quantification, the crossing point $(\mathrm{Cp})$ value of miR-30d or miR-107 was normalized to the $\mathrm{Cp}$ value of $\beta$-actin and $\mathrm{U} 6$ as a control. miR-30d sense, 5'-CTTTCAGTCAGATGTTT GCTGC-3' and antisense, 5'-ATTGCGTGTCGTGGAG TCG-3'; miR-107 sense, 5'-AGCAGCATTGTACAGGGCTA TCA-3' and antisense, 5'-ATTGCGTGTCGTGGAGTCG-3'; U6 sense, 5'-GCTTCGGCAGCACATATACTAAAAT-3' and antisense, 5'-CGCTTCACGAATTTGCGTGTCAT-3'.

Western blot analysis. Whole cell and tissue extracts were prepared in cell lysis buffer followed by immunoblotting with anti-HO-1 antibody $(1: 4,000), \beta$-actin $(1: 4,000)$, Akt $(1: 1,000)$, pAkt $(1: 1,000)$, ERK1/2 $(1: 1,000)$ and p-ERK1/2 $(1: 1,000)$ (Cell Signaling Technology) as previously described (3).

In vivo tumor growth assays. BALB/c-nu nude mice (aged 4 weeks, male) were purchased from the Shanghai Laboratory Animal Center (Shanghai, China). The Institutional Animal Care and Use Committee of Harbin Medical University approved all animal experiments. Mice housed under identical conditions were allowed free access to a standard diet and to tap water with a $12 \mathrm{~h}$ light: $12 \mathrm{~h}$ dark cycle. Four-week-old male nude mice were anaesthetized by barbital sodium at $70 \mathrm{mg} / \mathrm{kg}$ body weight and a laparotomy was performed. Ten mice were randomly divided into two groups. These two groups of mice were injected subcutaneously (s.c). with $1 \times 10^{6}$ stably transfected HepG2/HO-1 and HepG2/Mock cells in the left flank. After 19 days, the mice were sacrificed and photographed. Tumors were harvested for paraffin embedding, sectioning and histological examination after hematoxylin and eosin staining. Five animals were included in each group. Data are shown for representative experiments.

Statistical analysis. All data presented in the present study have been repeated at least three times from three independent experiments and are expressed as the mean \pm standard error. Student's t-test was performed to determine the significance of the respective group for each experimental test condition. $\mathrm{P}<0.05, \mathrm{P}<0.01$ or $\mathrm{P}<0.001$ indicated a significant difference.

\section{Results}

HO-1 modulates miRNA profile in HepG2 cells. In previous studies, we demonstrated that HO- 1 could inhibit the migratory ability of HepG2 cells (3). To further clarify the molecular mechanism of HO-1 as a tumor regulator, microarray was used to detect the difference of the miRNA profile between mouse HO-1 overexpressed HepG2 cells (H1B) and empty vector group (Mock). As results show (Fig. 1A), there are 19 miRNAs upregulated and 23 downregulated at least two-fold by HO-1 overexpression. Numerous downregulated miRNAs were also found to relate to tumor progression such as hsa-let-7 and hsa-miR-19b (Fig. 1A). Six miRNAs closely related to tumor 
A

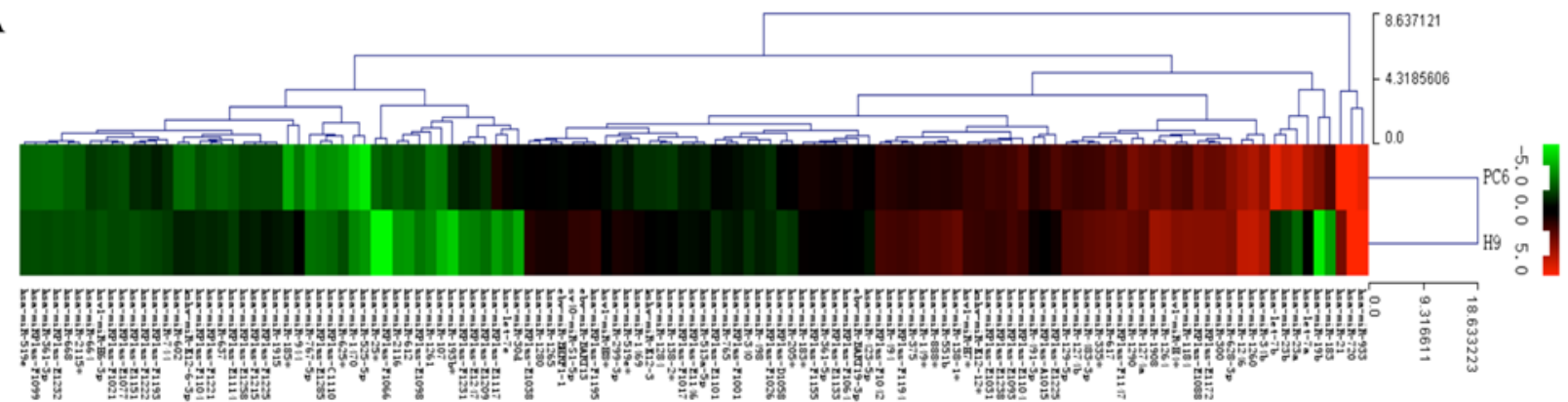

B

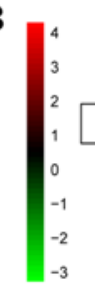

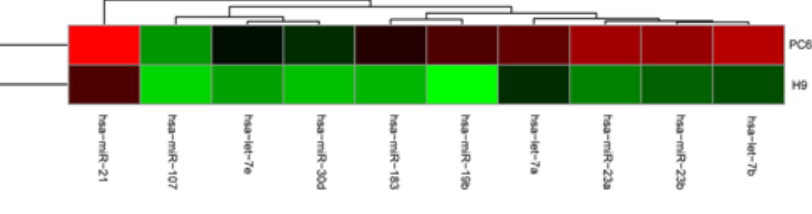

\begin{tabular}{|c|c|c|}
\hline ID & Name & H1B/Mock \\
\hline 145846 & hsa-let-7e & 0.127411669 \\
\hline 146011 & hsa-let-7a & 0.121024687 \\
\hline 10923 & hsa-miR-107 & 0.401045131 \\
\hline 19596 & hsa-miR-30d & 0.114042985 \\
\hline 10977 & hsa-miR-183 & 0.038519918 \\
\hline 42744 & hsa-miR-23a & 0.01452999 \\
\hline 145841 & hsa-miR-23b & 0.030220576 \\
\hline 10998 & hsa-miR-19b & 0.006976106 \\
\hline 5740 & hsa-miR-21 & 0.067037667 \\
\hline 17749 & hsa-let-7b & 0.02408741 \\
\hline
\end{tabular}

C

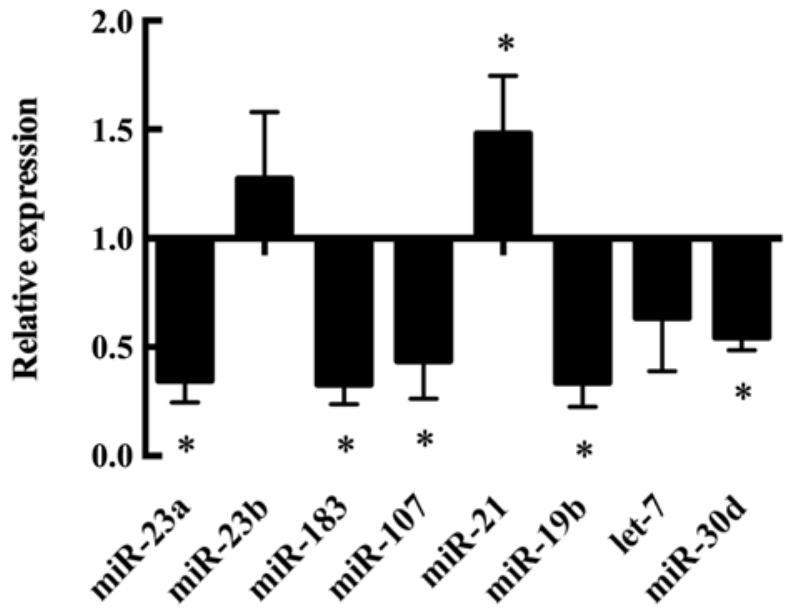

Figure 1. HO-1 modulates miRNAs expression in HO-1 overexpression HepG2 cells. (A) A heatmap of the miRNAs with significantly changed expression when overexpressing HO-1 compared with the Mock. Average expression is shown for each miRNA in each class. (B) The miRNAs closely related to tumor metastasis were chosen to show the details. (C) Quantitative RT-PCR analysis of these eight miRNAs in HO-1 overexpressing HepG2 cells (H1B) compared with the Mock (Mock); ${ }^{*} \mathrm{P}<0.05$ vs. the control.

progression were chosen to verify their expression by qPCR in H1B and Mock cells (Fig. 1B and C).

HO- 1 reduces the expression of $\mathrm{miR}-30 \mathrm{~d}$ and $\mathrm{miR}-107$ both in vitro and in vivo. In order to confirm the relation of $\mathrm{HO}-1$ and miRNA expression in human HCC, we altered the expression of HO-1 by genetic manipulation in HepG2 cells and detected the expression of HO-1 and above the six miRNA level. The results found that the abundance of miR-30d and miR-107 were stably negatively correlated with the expression of HO-1 (Fig. 2A and B). To pinpoint critical relation between HO-1 and miRNAs, we generated human HO-1 (hHO-1) stable overexpression HepG2 cell lines (named HH-3 and HH-6). The expression of HO-1 in the reconstituted HepG2 cell lines was verified by western blotting (Fig. 2C). Next, we detected the expression of HO-1 and miR-30d/miR-107 in HH-3 and HH-6. The results show that the expression of miR-30d and miR-107 were stably decreased (Fig. 2C). Furthermore, we detected the expression of $\mathrm{miR}-30 \mathrm{~d} / \mathrm{miR}-107$ in the livers of HO-1 Tg mice (Fig. 2D). All results showed that HO-1 and $\mathrm{miR}-30 \mathrm{~d} / \mathrm{miR}-107$ expressions were negatively correlated.
Moreover, the HO-1 and $\mathrm{miR}-30 \mathrm{~d} / \mathrm{miR}-107$ expression were also analyzed after treating cells by $\mathrm{HO}-1$ inducer cobalt protoporphyrin IX (CoPPIX) or HO-1 activity suppressor Sn protoporphyrin IX (SnPP). First, we confirmed the induction of HO-1 by CoPPIX was both dose- and time-dependent (Fig. 3A). Notably, we found the regulatory role of $\mathrm{HO}-1$ in $\mathrm{miR}-30 \mathrm{~d} / \mathrm{miR}-107$ expression was also time-dependent (Fig. 3B and C). Moreover, the variation of miR-30d/miR-107 after SnPPIX exposure was detected. The results showed SnPPIX treatment restored the miR-30d/ miR-107 expression (Fig. 3D and E). Considering SnPPIX blocks the enzymatic activity of HO-1 without influencing its expression, this suppressive effect of HO-1 on miR-30d/ miR-107 may be due to HO-1 activity.

HO-1 overexpression reduces the expression of miR-30d and miR-107 via its metabolite iron. Although some researchers have found out new functions of HO-1, we could not neglect that the main role of HO-1 is still as an enzyme functioning through its active products. Our results also implied that HO-1 may regulate the level of miR-30d/miR-107 by its metabolites. 
A
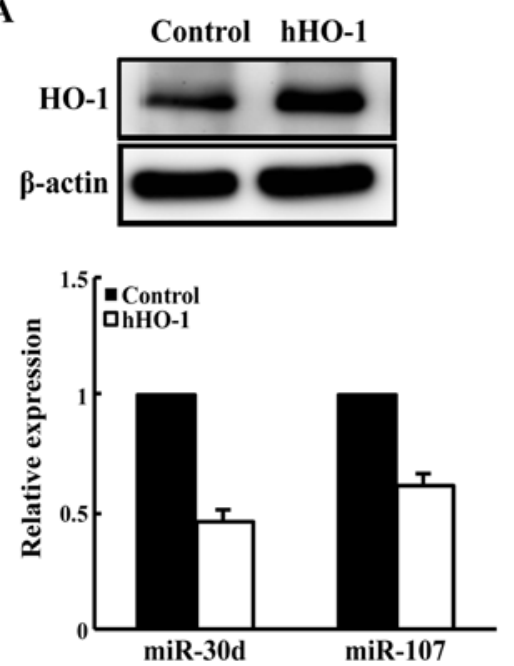

C

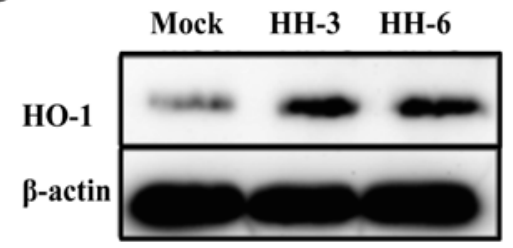

B
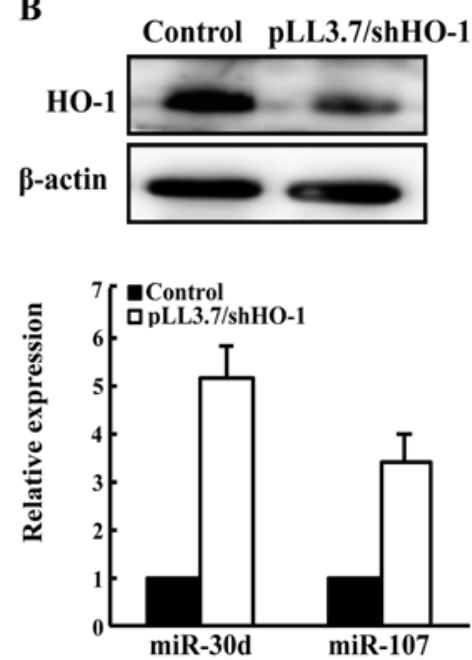

D

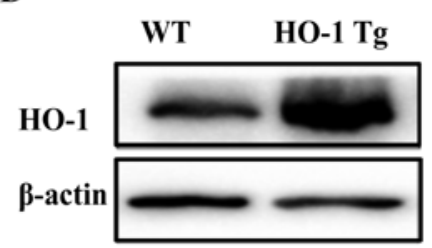

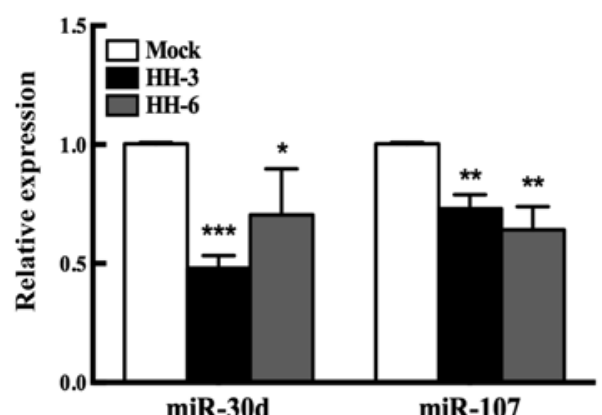

miR-30d

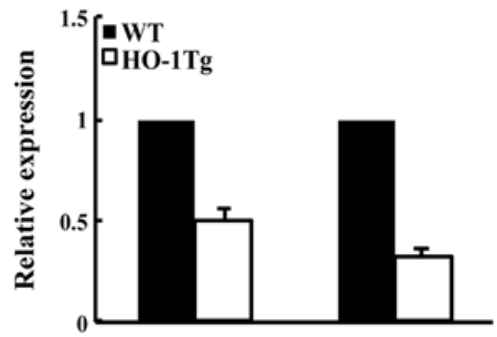

miR-30d

miR-107

Figure 2. HO-1 overexpression downregulates miR-30d/miR-107. (A) Western blot and quantitative RT-PCR analyses were performed to analyze HO-1 and miR-30d/miR-107 expression in HepG2 cells by gene manipulation: (A) overexpressed human HO-1 (hHO-1); (B) interference of the expression of HO-1 by pLL3.7/shHO-1; (C) The HO-1 protein expression and miR-30d/miR-107 mRNA level were evaluated in stable overexpressed HO-1 cell lines HH-3 and HH-6 and (D) HO-1 Tg mouse livers; ${ }^{*} \mathrm{P}<0.05,{ }^{* *} \mathrm{P}<0.01,{ }^{* * * *} \mathrm{P}<0.001$ vs. the control.

To determine which product of HO-1 activity could be responsible for $\mathrm{miR}-30 \mathrm{~d} / \mathrm{miR}-107$ downregulation, we treated HepG2 cells with ferricitrate, deferoxamine, $\mathrm{CO}$-releasing molecule (CORM), inactive CORM (iCORM) and bilirubin (Fig. 3F-H). It turned out that the effect of HO-1 on $\mathrm{miR}-30 \mathrm{~d} / \mathrm{miR}-107$ could be mimicked by only one of its products - iron. Moreover, the opposite results can be received by treating deferoxamine (Fig. 3H). These results demonstrated that iron maybe an important mediator in the procedure of HO-1 regulating $\mathrm{miR}-30 \mathrm{~d} / \mathrm{miR}-107$.

HO-1 inhibits the proliferation by suppressing miR-30d and $m i R-107$. To elucidate whether $\mathrm{miR}-30 \mathrm{~d} / \mathrm{miR}-107$ is involved in the function of HO-1 in HCC, MTT and colony formation assays were performed different manipulations in HCC cells. MTT results showed that the numbers of viable cells in HH-3 and HH6 were significantly fewer than that of
Mock cells (Fig. 4A). Opposite results were found through transient transfection of HepG2 cells with miR-30d/miR-107 mimics (Fig. 4B). Colony formation assays also showed that HO-1 overexpression could inhibit the proliferation of HCC cells (Fig. 4C). In vivo, we observed the growth rate of HepG2 cells subcutaneous xenografts. Compared with the Mock, the tumor size in HH3 and HH6 group showed a marked reduction (Fig. 4D and E).

The results also showed that the beneficial effect of HO-1 on HCC proliferation could be partially reversed by increasing the expression of miR-30d and miR-107 (Fig. 4F and G). These data demonstrated that HO-1 suppressed the proliferative ability of HepG2 cells via inhibiting the expression of miR-30d/miR-107.

miR-30d/miR-107 is an important mediator in antimetastasis function of HO-1. To investigate whether human HO-1 could 
A

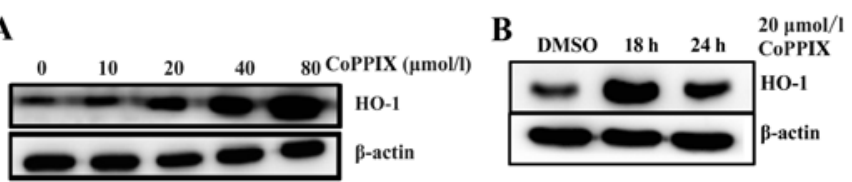

D

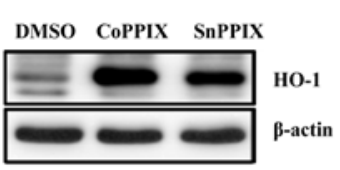

F

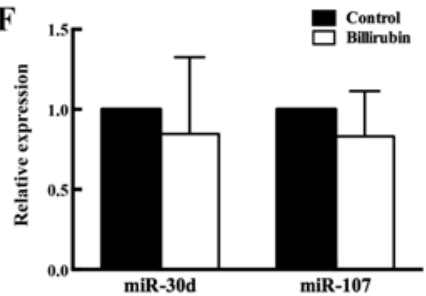

E

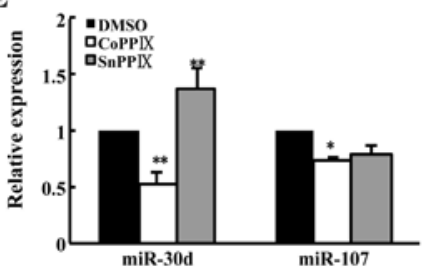

G

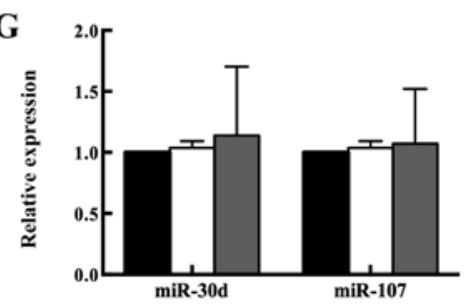

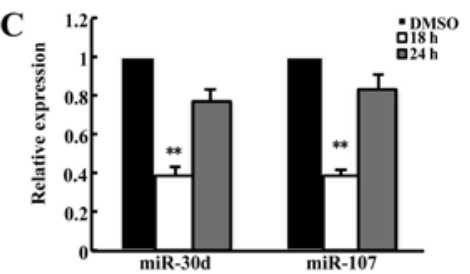

H

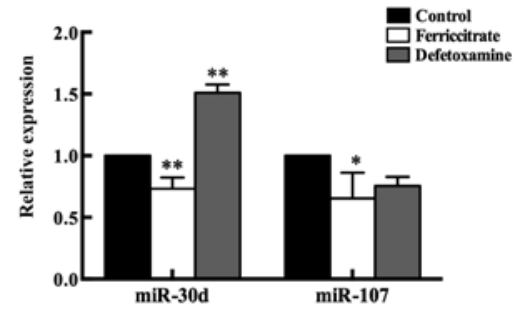

rriccitrate

Control

吕 CORM

\section{.}


A

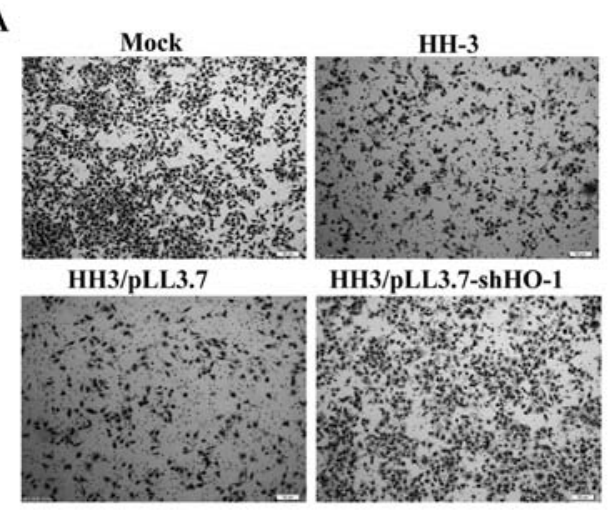

C

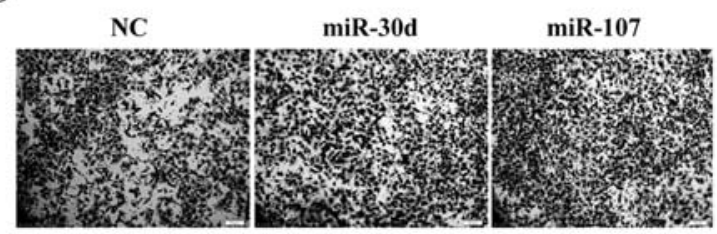

D

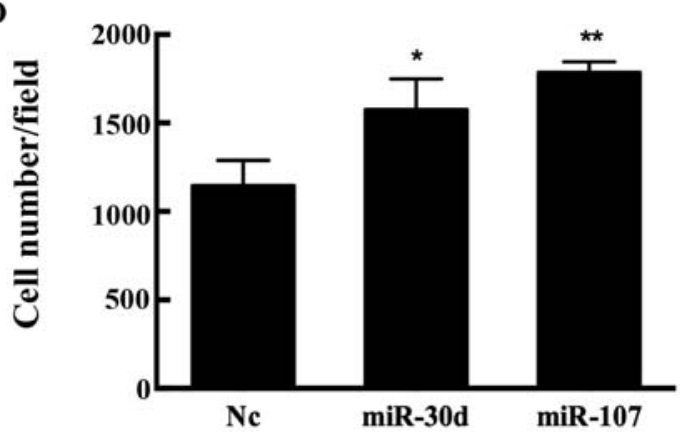

B

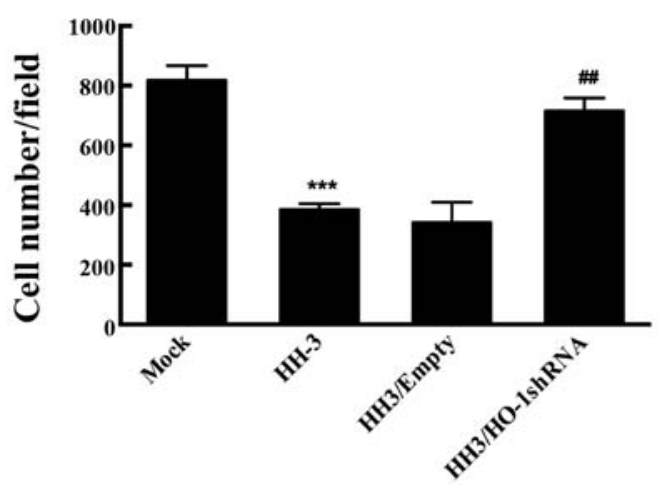

E

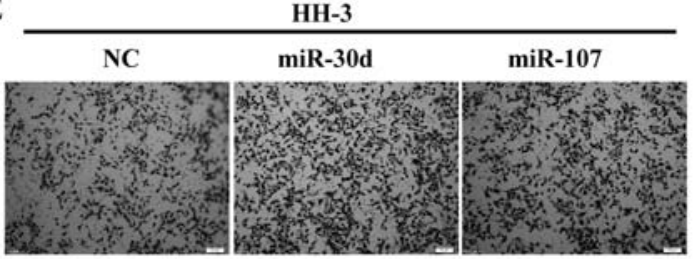

F

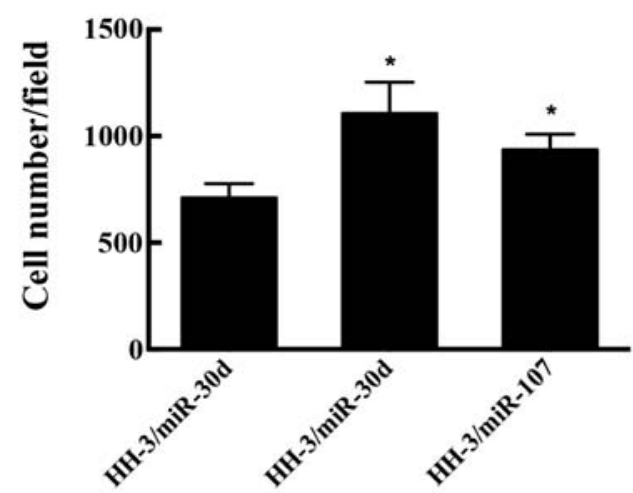

Figure 5. HO-1 and miR-30d/miR-107 regulate the migration of HepG2 cells. (A-B) Mock and HH-3 cells were transfected with the indicated plasmid for $24 \mathrm{~h}$, and plated 50,000 cells/well on Transwell inserts and allowed to migrate for $24 \mathrm{~h}$ toward media with $10 \%$ fetal bovine serum. (A) Representative images showing the cell density on the filter, bottom (graph), and (B) quantitative analysis for the cells migrating through the filter in three independent experiments. (C and D) HepG2 cells were transfected with the indicated oligonucleotides for $24 \mathrm{~h}$, plated 50,000 cells/well were on Transwell inserts to perform the Transwell assays. Top, (C) representative images showing the cell density on the filter, and (D) quantitative analysis for the cells migrating through the filter in three independent experiments. (E and F) HH-3 cells were respectively transfected by the indicated oligonucleotides for $24 \mathrm{~h}$ and following the migration assay. (E) Top, representative images showing the cell density on the filter, bottom (graph), and (F) quantitative analysis for the cells migrating through the filter in three independent experiments; ${ }^{*} \mathrm{P}<0.05,{ }^{* *} \mathrm{P}<0.01,{ }^{* * * *} \mathrm{P}<0.001$.

inhibit metastasis of HCC, similarly to mouse HO-1, we observed the migratory ability of HH3 and HH6 by Transwell assays. The results confirmed that hHO-1 could inhibit the migration of HepG2 cells. HO-1 silencing enhanced the migration of HepG2 cells (Fig. 5A and B). As known, miR-30d is a promoter of metastasis in Huh7 cells (16). Moreover, we demonstrated miR-107 could accelerate the migration of HCC cells (Fig. 5C and D) (3). Our further studies confirmed that hHO-1 anti-migration effect could also be reversed by overexpression of miR-30d and miR-107 (Fig. 5E and F). Therefore, we demonstrated that HO-1 could suppress the migration of HCC by decreasing miR-30d/miR-107 expression.

HO-1/miR-30d/miR-107 regulate the HCC progression through PI3K/AKT and MAPK/ERK pathways. According to the above studies, we elucidated that the proliferative and metastatic capability of HepG2 cells was regulated by HO-1 and miR-30/miR-107 in backward directions. To further investigate the specific factors involved in the regulation, we transfected HepG2 cells with pcDNA3.1 (+)-hHO-1 or the mimics of $\mathrm{miR}-30 \mathrm{~d} / \mathrm{miR}-107$ to measure the activity of Akt and ERK1/2 pathways. The results identified that HO-1 overexpression could decrease the phosphorylation of AKT and ERK. miR-30d/miR-107 showed a crosscurrent in the regulation of the above factors (Fig. 6A). These results suggested that Akt and ERK pathways could be involved in the function of HO-1/miR-30d/miR-107 axis (Fig. 6B).

\section{Discussion}

Heme oxygenase-1 (HO-1) is an inducible enzyme catalyzing the first rate-limiting step in degradation of heme and playing an important role in many pathophysiological processes. HO-1 gene polymorphism leads to a correlation with cancer 

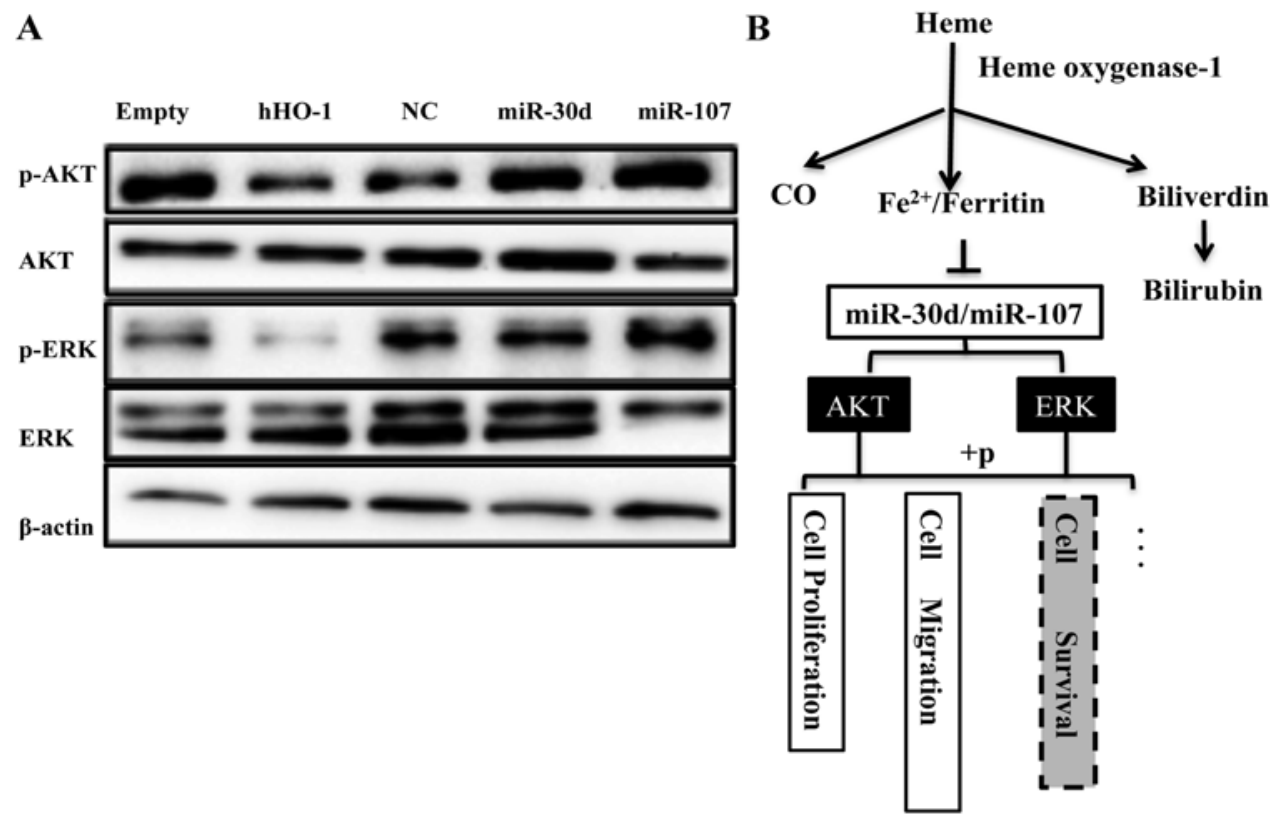

Figure 6. HO-1 and miR-30d/miR-107 modulate the HCC through PI3K/AKT and MAPK/ERK. (A) HepG2 cells were transfected as indicated plasmid and oligonucleotides. The protein levels of pAKT, AKT, p-ERK and ERK were detected by western blotting. $\beta$-actin were used as reference. (B) The pathway map for the present study is shown.

susceptibility (17-19). Elevated HO-1 has been detected in various tumors, including adenocarcinoma, glioblastoma, melanoma, prostate and pancreatic cancer, thereby affecting tumor cell apoptosis, proliferation, invasion and metastasis $(2,3)$. Notably, HO-1 has shown its influence on tumors is cell-type specific and can be the opposite in different tissues (2). The mechanism of this complicated character of HO-1 has not been clarified.

Recently, accumulated evidence demonstrates that heme metabolism system is involved in microRNA biogenesis. Firstly, Faller et al found that heme is involved in pri-miRNA processing via promoting dimerization of DGCR 8 which is essential for the first step of miRNA processing (20). Subsequently, Li et al verified that iron homeostasis regulates the activity of the microRNA pathway through poly(C)binding protein 2 (21). Several studies also observed different interaction between HO-1 and miRNAs in different tissues. For example, Skrzypek et al confirmed HO-1 could inhibit growth, vascularization and distal metastasis through its regulation on miR-378 in non-small cell lung carcinoma (22). Moreover, Kozakowska et al attested that HO-1 inhibits myoblast differentiation by targeting several myo-miRNAs (23). These results showed that the regulation of HO-1 on miRNAs is tissuespecific. In the present study, we investigated the relationship between HO-1 and miR-30d/miR-107 and its mechanism in hepatocellular carcinoma.

The mechanism of HO-1 regulating miRNAs is disputable. In myoblasts, $\mathrm{HO}-1$ regulates the miRNA biogenesis by suppressing the DGCR8 level (23). Overexpression of HO-1 in NCI-H292 lung cancer cells upregulates DGCR8 and Drosha (22). DGCR8 and Drosha are important microprocessors in regulating the homeostasis of miRNAs pool. However, in the present study we found out that HO-1 only impacted on several specific miRNA levels in HCC. The disturbance of DGCR8 and Drosha expression or the fluctuation of microRNA pool cannot explain these results. Considering the classic role of HO-1, the catalytic activity, its catalysates of heme could be the important candidates in the regulation between HO-1 and miRNAs. In a previous study, $\mathrm{CO}$ has been demonstrated as an important mediator in the interplay between HO-1 and miR-378 in lung cancer (22). In the present study, our results showed that another metabolite product, iron, could mimic the inhibitory effect of HO-1 on miR-30d/ miR-107. Furthermore, this effect could be reversed by deferoxamine treatment. Other products including $\mathrm{CO}$ and bilirubin did not mimic the HO-1 effect on miR-30d/miR-107.

Furthermore, we also noted that miR-107 expression was difficult to be upregulated significantly by SnPP or deferoxamine, unlike miR-30d. Previous research reported that miR-107 could directly target Dicer (24), which is a key enzyme of miRNAs processing. There may be a feedback regulation between miR-107 and Dicer, which could be the reason for the limited upregulation.

Growing number of studies show that the role of miR-30d and miR-107 is cell or tissue-specific, which may be dependent on cellular context or different downstream target genes $(16,25)$. A previous study, we firstly reported that miR-107 played a pro-proliferation and pro-metastasis role in HCC (26). In addition, consistent with Yao et al (16), we also confirmed that miR-30d promoted the progress of liver cancer. In the present study, the results showed when overexpressing HO-1, with transfected miR-30d/miR-107 mimic, the benefit of HO-1 overexpression on HCC inhibition may be counteracted. These data confirmed that HO-1 restrains the HCC proliferation and migration depending partly on downregulating $\mathrm{miR}-30 \mathrm{~d} / \mathrm{miR}-107$. Moreover, the specific pathway for the regulation needs to be clarified.

The phosphoinositide 3-kinase (PI3K)/Akt and extracellular signal-regulated kinase (ERK) pathways are important for many biological processes. Activation of PI3K/AKT and 
MAPK/ERK pathways via phosphorylation of their variety substrates are widely known to promote cell proliferation, survival, apoptosis and cell migration $(27,28)$. In the present study, we showed that HO-1 could inhibit the phosphorylation of Akt and ERK, which may lead to a suppression of the proliferation and migration of HCC. Moreover, miR-30d and miR-107 could activate these signaling pathways. These results together suggested that PI3K/Akt and MAPK/ERK pathways maybe involved in the modulation of HO-1/miR-30d/miR-107 in cancer progress.

In conclusion, the present study uncovers a new mechanism of HO-1 function depending on the regulation of miRNAs in HCC. HO-1 acts as a tumor-suppressor via downregulating the expression of miR-30d/miR-107. The catalytic products of HO-1 play important roles in this regulation. Moreover, PI3K/Akt and MAPK/ERK signals may be the final effective pathway.

\section{Acknowledgements}

The present study was supported by the Natural Science Foundation of China (81171997/81572347), the China Postdoctoral Science Foundation (2015M581479), the Heilongjiang Postdoctoral Science Foundation (LBH-Z15125), and the Natural Science Foundation of Heilongjiang Province for youth (QC2011C016).

\section{References}

1. Maines MD, Trakshel GM and Kutty RK: Characterization of two constitutive forms of rat liver microsomal heme oxygenase. Only one molecular species of the enzyme is inducible. J Biol Chem 261: 411-419, 1986.

2. Jozkowicz A, Was H and Dulak J: Heme oxygenase-1 in tumors: Is it a false friend? Antioxid Redox Signal 9: 2099-2117, 2007.

3. Zou C, Zhang H, Li Q, Xiao H, Yu L, Ke S, Zhou L, Liu W, Wang W, Huang H, et al: Heme oxygenase-1: A molecular brake on hepatocellular carcinoma cell migration. Carcinogenesis 32 : 1840-1848, 2011

4. Deininger MH, Meyermann R, Trautmann K, Duffner F, Grote EH, Wickboldt J and Schluesener HJ: Heme oxygenase (HO)-1 expressing macrophages/microglial cells accumulate during oligodendroglioma progression. Brain Res 882: 1-8, 2000.

5. Doi K, Akaike T, Fujii S, Tanaka S, Ikebe N, Beppu T, Shibahara S, Ogawa $\mathrm{M}$ and Maeda $\mathrm{H}$ : Induction of haem oxygenase-1 nitric oxide and ischaemia in experimental solid tumours and implications for tumour growth. Br J Cancer 80: 1945-1954, 1999.

6. De Palma G, Mozzoni P, Acampa O, Internullo E, Carbognani P, Rusca M, Goldoni M, Corradi M, Tiseo M, Apostoli P, et al: Expression levels of some antioxidant and epidermal growth factor receptor genes in patients with early-stage non-small cell lung cancer. J Nucleic Acids 2010: pii: 147528, 2010.

7. Lee SE, Yang H, Jeong SI, Jin YH, Park CS and Park YS: Induction of heme oxygenase-1 inhibits cell death in crotonaldehyde-stimulated HepG2 cells via the PKC- $\delta$-p38-Nrf2 pathway. PLoS One 7: e41676, 2012.

8. Kim DH, Kim JH, Kim EH, Na HK, Cha YN, Chung JH and Surh YJ: 15-Deoxy-Delta12,14-prostaglandin J2 upregulates the expression of heme oxygenase-1 and subsequently matrix metalloproteinase-1 in human breast cancer cells: Possible roles of iron and ROS. Carcinogenesis 30: 645-654, 2009.

9. Lin CW, Shen SC, Hou WC, Yang LY and Chen YC: Heme oxygenase-1 inhibits breast cancer invasion via suppressing the expression of matrix metalloproteinase-9. Mol Cancer Ther 7: 1195-1206, 2008.

10. Liu PL, Tsai JR, Charles AL, Hwang JJ, Chou SH, Ping YH, Lin FY, Chen YL, Hung CY, Chen WC, et al: Resveratrol inhibits human lung adenocarcinoma cell metastasis by suppressing heme oxygenase 1-mediated nuclear factor-kappaB pathway and subsequently downregulating expression of matrix metalloproteinases. Mol Nutr Food Res 54 (Suppl 2): S196-S204, 2010.
11. Heo I and Kim VN: Regulating the regulators: Posttranslational modifications of RNA silencing factors. Cell 139: 28-31, 2009.

12. Beckman JD, Chen C, Nguyen J, Thayanithy V, Subramanian S, Steer CJ and Vercellotti GM: Regulation of heme oxygenase-1 protein expression by miR-377 in combination with miR-217. J Biol Chem 286: 3194-3202, 2011.

13. Qiu L, Fan H, Jin W, Zhao B, Wang Y, Ju Y, Chen L, Chen Y, Duan $Z$ and Meng S: miR-122-induced down-regulation of HO-1 negatively affects miR-122-mediated suppression of HBV. Biochem Biophys Res Commun 398: 771-777, 2010.

14. Shan Y, Zheng J, Lambrecht RW and Bonkovsky HL: Reciprocal effects of micro-RNA-122 on expression of heme oxygenase-1 and hepatitis $\mathrm{C}$ virus genes in human hepatocytes. Gastroenterology 133: 1166-1174, 2007.

15. Kozakowska M, Szade K, Dulak J and Jozkowicz A: Role of heme oxygenase-1 in postnatal differentiation of stem cells: A possible cross-talk with microRNAs. Antioxid Redox Signal 20: 1827-1850, 2014.

16. Yao J, Liang L, Huang S, Ding J, Tan N, Zhao Y, Yan M, Ge C, Zhang Z, Chen T, et al: MicroRNA-30d promotes tumor invasion and metastasis by targeting Galphai 2 in hepatocellular carcinoma. Hepatology 51: 846-856, 2010.

17. Chin HJ, Cho HJ, Lee TW, Na KY, Yoon HJ, Chae DW, Kim S, Jeon US, Do JY, Park JW, et al; Progressive REnal disease and Medical Informatics and gEnomics Research (PREMIER) members: The heme oxygenase-1 genotype is a risk factor to renal impairment of $\operatorname{IgA}$ nephropathy at diagnosis, which is a strong predictor of mortality. J Korean Med Sci 24 (Suppl 1): S30-S37, 2009

18. Sunamura M, Duda DG, Ghattas MH, Lozonschi L, Motoi F, Yamauchi J, Matsuno S, Shibahara S and Abraham NG: Heme oxygenase-1 accelerates tumor angiogenesis of human pancreatic cancer. Angiogenesis 6: 15-24, 2003.

19. Vashist YK, Blessmann M, Trump F, Kalinin V, Kutup A, Schneider C, Gawad K, Kaifi JT, Schmelzle R, Izbicki JR, et al: Microsatellite GTn-repeat polymorphism in the promoter of heme oxygenase-1 gene is an independent predictor of tumor recurrence in male oral squamous cell carcinoma patients. J Oral Pathol Med 37: 480-484, 2008.

20. Faller M, Matsunaga M, Yin S, Loo JA and Guo F: Heme is involved in microRNA processing. Nat Struct Mol Biol 14: 23-29, 2007.

21. Li Y, Lin L, Li Z, Ye X, Xiong K, Aryal B, Xu Z, Paroo Z, Liu Q, He C, et al: Iron homeostasis regulates the activity of the microRNA pathway through poly(C)-binding protein 2 . Cell Metab 15: 895-904, 2012.

22. Skrzypek K, Tertil M, Golda S, Ciesla M, Weglarczyk K, Collet G, Guichard A, Kozakowska M, Boczkowski J, Was H, et al: Interplay between heme oxygenase-1 and miR-378 affects non-small cell lung carcinoma growth, vascularization, and metastasis. Antioxid Redox Signal 19: 644-660, 2013.

23. Kozakowska M, Ciesla M, Stefanska A, Skrzypek K, Was H, Jazwa A, Grochot-Przeczek A, Kotlinowski J, Szymula A, Bartelik A, et al: Heme oxygenase-1 inhibits myoblast differentiation by targeting myomirs. Antioxid Redox Signal 16: 113-127, 2012.

24. Martello G, Rosato A, Ferrari F, Manfrin A, Cordenonsi M, Dupont S, Enzo E, Guzzardo V, Rondina M, Spruce T, et al: A MicroRNA targeting dicer for metastasis control. Cell 141: 1195-1207, 2010.

25. Finnerty JR, Wang WX, Hébert SS, Wilfred BR, Mao G and Nelson PT: The miR-15/107 group of microRNA genes: Evolutionary biology, cellular functions, and roles in human diseases. J Mol Biol 402: 491-509, 2010.

26. Zou CD, Zhao WM, Wang XN, Li Q, Huang H, Cheng WP Jin JF, Zhang H, Wu MJ, Tai S, et al: MicroRNA-107: A novel promoter of tumor progression that targets the CPEB3/EGFR axis in human hepatocellular carcinoma. Oncotarget 7: 266-278, 2016.

27. Cheng P, Alberts I and Li X: The role of ERK1/2 in the regulation of proliferation and differentiation of astrocytes in developing brain. Int J Dev Neurosci 31: 783-789, 2013.

28. Fu J, Lv H, Guan H, Ma X, Ji M, He N, Shi B and Hou P. Metallothionein $1 \mathrm{G}$ functions as a tumor suppressor in thyroid cancer through modulating the PI3K/Akt signaling pathway. BMC Cancer 13: 462, 2013. 